This item was submitted to Loughborough's Research Repository by the author.

Items in Figshare are protected by copyright, with all rights reserved, unless otherwise indicated.

\title{
On a class of integrable systems of Monge-Ampere type
}

PLEASE CITE THE PUBLISHED VERSION

https://doi.org/10.1063/1.4984982

PUBLISHER

AIP Publishing

VERSION

AM (Accepted Manuscript)

\section{PUBLISHER STATEMENT}

This work is made available according to the conditions of the Creative Commons Attribution-NonCommercialNoDerivatives 4.0 International (CC BY-NC-ND 4.0) licence. Full details of this licence are available at: https://creativecommons.org/licenses/by-nc-nd/4.0/

\section{LICENCE}

CC BY-NC-ND 4.0

\section{REPOSITORY RECORD}

Doubrov, B., E.V. Ferapontov, B. Kruglikov, and V.S. Novikov. 2019. "On a Class of Integrable Systems of Monge-ampere Type”. figshare. https://hdl.handle.net/2134/25223. 


\title{
On a class of integrable systems of Monge-Ampère type
}

\author{
B. Doubrov ${ }^{1}$, E.V. Ferapontov ${ }^{2}$, B. Kruglikov ${ }^{3,4}$, V.S. Novikov ${ }^{2}$ \\ ${ }^{1}$ Department of Mathematical Physics \\ Faculty of Applied Mathematics \\ Belarussian State University \\ Nezavisimosti av. 4, 220030 Minsk, Belarus \\ ${ }^{2}$ Department of Mathematical Sciences \\ Loughborough University \\ Loughborough, Leicestershire LE11 3TU \\ United Kingdom \\ ${ }^{3}$ Department of Mathematics and Statistics \\ UiT the Arctic University of Norway \\ Tromsø 90-37, Norway \\ ${ }^{4}$ Department of Mathematics and Natural Sciences \\ University of Stavanger, 40-36 Stavanger, Norway \\ e-mails: \\ doubrov@islc.org \\ E.V.Ferapontov@lboro.ac.uk \\ boris.kruglikov@uit.no \\ V.Novikov@lboro.ac.uk
}

\begin{abstract}
We investigate a class of multi-dimensional two-component systems of Monge-Ampère type that can be viewed as generalisations of heavenly-type equations appearing in self-dual Ricci-flat geometry. Based on the Jordan-Kronecker theory of skew-symmetric matrix pencils, a classification of normal forms of such systems is obtained. All two-component systems of Monge-Ampère type turn out to be integrable, and can be represented as the commutativity conditions of parameter-dependent vector fields.

Geometrically, systems of Monge-Ampère type are associated with linear sections of the Grassmannians. This leads to an invariant differential-geometric characterisation of the Monge-Ampère property.

MSC: 35F20, 35Q75, 37K10, 37K25, 53B50, 53Z05.
\end{abstract}

Keywords: System of Monge-Ampère type, heavenly-type equation, skew-symmetric matrix pencil, Jordan-Kronecker normal form, dispersionless Lax representation, linear section of the Grassmannian. 


\section{Introduction}

Let $u(\mathbf{x})$ and $v(\mathbf{x})$ be functions of $d$ independent variables $\mathbf{x}=\left(x^{1}, \ldots, x^{d}\right)$. In paper [6] we have initiated the study of integrability of first-order systems of the form

$$
F\left(u_{1}, \ldots, u_{d}, v_{1}, \ldots, v_{d}\right)=0, \quad G\left(u_{1}, \ldots, u_{d}, v_{1}, \ldots, v_{d}\right)=0,
$$

where $F, G$ are (nonlinear) functions of the partial derivatives $u_{i}=\frac{\partial u}{\partial x^{i}}, v_{i}=\frac{\partial v}{\partial x^{i}}$. The geometry behind systems $(1)$ is as follows. Let $V$ be the $(d+2)$-dimensional vector space with coordinates $x^{1}, \ldots, x^{d}, u, v$. Solutions to system (1) correspond to $d$-dimensional submanifolds of $V$ defined as $u=u(\mathbf{x}), v=v(\mathbf{x})$. Their $d$-dimensional tangent spaces, specified by the equations $d u=u_{i} d x^{i}, d v=v_{i} d x^{i}$, are parametrised by $2 \times d$ matrices

$$
U=\left(\begin{array}{lll}
u_{1} & \cdots & u_{d} \\
v_{1} & \cdots & v_{d}
\end{array}\right)
$$

whose entries are restricted by equations (1). Thus, equations (1) can be interpreted as the defining equations of a codimension two submanifold $X$ in the $\operatorname{Grassmannian} \operatorname{Gr}(d, V)$. Solutions to system (1) correspond to $d$-dimensional submanifolds of $V$ whose tangent spaces (translated to the origin) are contained in $X$. Equations of type (1) arise in numerous applications in the theory of dispersionless integrable systems, general relativity and differential geometry. For $d=3$ their integrability aspects, as well as the geometry of the associated fourfolds $X \subset \mathbf{G r}(3,5)$, were thoroughly investigated in [6].

In this paper we consider an important subclass of multi-dimensional $(d \geq 3)$ equations (1) known as systems of Monge-Ampère type (Jacobi systems),

$$
\begin{aligned}
& a^{i j}\left(u_{i} v_{j}-u_{j} v_{i}\right)+b^{i} u_{i}+c^{i} v_{i}+m=0, \\
& \alpha^{i j}\left(u_{i} v_{j}-u_{j} v_{i}\right)+\beta^{i} u_{i}+\gamma^{i} v_{i}+\mu=0,
\end{aligned}
$$

where each equation corresponds to a constant-coefficient linear combination of the minors of $U$. Systems of type (2) were discussed previously in [3] from the point of view of 'complete exceptionality' of the Cauchy problem. Geometrically, submanifolds $X$ associated with such systems are linear sections of the Plücker embedding of $\mathbf{G r}(d, V)$ into $\mathbb{P} \Lambda^{d}(V)$. Note that the class of Monge-Ampère systems is invariant under the natural action of the equivalence group $\mathbf{S L}(V)$. In what follows we assume systems (1), (2) to be non-degenerate in the sense that the corresponding characteristic variety,

$$
\operatorname{det}\left[\sum_{i=1}^{d} p_{i}\left(\begin{array}{cc}
F_{u_{i}} & F_{v_{i}} \\
G_{u_{i}} & G_{v_{i}}
\end{array}\right)\right]=0,
$$

defines an irreducible quadric of rank $\mathrm{d}$ for $d \leq 4$, and rank 4 for $d>4$ (note that 4 is the maximal possible value for the rank of a quadratic form representable as the determinant of a $2 \times 2$ matrix with entries linear in $p_{i}$ ). This non-degeneracy property holds for all examples of physical/geometric relevance.

Our main results can be summarised as follows:

- All Monge-Ampère systems (2) are integrable, with Lax representations in parameter-dependent commuting vector fields. This result was, in a sense, unexpected: indeed, it was demonstrated in [5] that second-order analogues of systems (1), known as symplectic Monge-Ampère equations, are not integrable in general for $d \geq 3$. Our approach is based on the observation that every MongeAmpère system (2) can be defined by a pair of differential $d$-forms in $V$, that is, by two elements of $\Lambda^{d}\left(V^{*}\right)$. Utilising the $\mathbf{S L}(V)$-equivariant duality between $\Lambda^{d}\left(V^{*}\right)$ and $\Lambda^{2}(V)$ we can reduce the theory of normal forms of Monge-Ampère systems to the classification of pencils of skew-symmetric two-forms. This, however, is the classical territory (in Sect. 2.1 we recall the main ingredients of the theory of Jordan-Kronecker normal forms of skew-symmetric matrix pencils). Thus we obtain normal forms of Monge-Ampère systems in all dimensions $d$ (see below), for which the integrability can be established directly.

- For $d=2,3$ any non-degenerate system of Monge-Ampère type is linearisable (Theorem 1 of Sect. 2.2). For $d=2$ this is certainly a well-known result, see e.g. [14]. 
- For $d=4$ any non-degenerate system of Monge-Ampère type is SL(6)-equivalent to one of the following normal forms (Theorem 2 of Sect. 2.3):

1. $u_{2}-v_{1}=0, \quad u_{3}+v_{4}=0$,

2. $u_{2}-v_{1}=0, \quad u_{3}+v_{4}+u_{1} v_{2}-u_{2} v_{1}=0$,

3. $u_{2}-v_{1}=0, \quad u_{3} v_{4}-u_{4} v_{3}-1=0$,

4. $u_{2}-v_{1}=0, \quad u_{1}+v_{2}+u_{3} v_{4}-u_{4} v_{3}=0$,

see Sect. 2.3 for the associated Lax representations. Introducing a potential $w$ such that $w_{1}=$ $u, w_{2}=v$, one obtains well-known integrable second-order PDEs: $w_{13}+w_{24}=0$ (linear equation), $w_{13}+w_{24}+w_{11} w_{22}-w_{12}^{2}=0$ (second heavenly equation [17]), $w_{13} w_{24}-w_{14} w_{23}-1=0$ (first heavenly equation [17]), and $w_{11}+w_{22}+w_{13} w_{24}-w_{14} w_{23}=0$ (Husain equation [13]), respectively. All of them originate from self-dual Ricci-flat geometry.

- For $d=5$ any non-degenerate system of Monge-Ampère type is SL(7)-equivalent to one of the following normal forms (Theorem 3 of Sect. 2.4):

1. $u_{1}+v_{2}+u_{3} v_{4}-u_{4} v_{3}=0, \quad u_{2}+v_{3}+u_{4} v_{5}-u_{5} v_{4}=0$,

2. $u_{2}-v_{1}=0, \quad u_{1}+v_{5}+u_{3} v_{4}-u_{4} v_{3}=0$,

3. $u_{2}-v_{1}=0, u_{4}+v_{5}+u_{1} v_{3}-u_{3} v_{1}=0$,

4. $u_{2}-v_{1}=0, \quad u_{5}+u_{3} v_{4}-u_{4} v_{3}=0$,

see Sect. 2.4 for the associated Lax representations. Note that most of the above normal forms (apart from case $1, d=5$ ) can be obtained as travelling wave reductions of the 6-dimensional integrable Monge-Ampère system

$$
u_{2}-v_{1}=0, \quad u_{5}+v_{6}+u_{3} v_{4}-u_{4} v_{3}=0,
$$

which reduces to the second-order equation $w_{15}+w_{26}+w_{13} w_{24}-w_{14} w_{23}=0$ for a potential $w$ defined as $w_{1}=u, w_{2}=v$. This equation appeared in hyper-Kähler geometry [19] and can be obtained as a reduction of $\operatorname{sdiff}\left(\Sigma^{2}\right)$ self-dual Yang-Mills equations [18].

- For arbitrary d generic normal forms are discussed in Sect. 2.5. Note that the cases of even/odd dimensions lead to essentially different normal forms. Thus, for even $d=2 k+2$ (Jordan case) a generic Monge-Ampère system can be reduced to the form

$$
\begin{gathered}
u_{2 k+1}=\left(u_{1} v_{2}-u_{2} v_{1}\right)+\left(u_{3} v_{4}-u_{4} v_{3}\right)+\ldots+\left(u_{2 k-1} v_{2 k}-u_{2 k} v_{2 k-1}\right), \\
v_{2 k+2}=a_{1}\left(u_{1} v_{2}-u_{2} v_{1}\right)+a_{2}\left(u_{3} v_{4}-u_{4} v_{3}\right)+\ldots+a_{k}\left(u_{2 k-1} v_{2 k}-u_{2 k} v_{2 k-1}\right),
\end{gathered}
$$

here $a_{1}, \ldots, a_{k}$ are arbitrary constants. For odd $d=2 k+1$ (Kronecker case) a generic MongeAmpère system can be reduced to the form

$$
\begin{aligned}
& u_{1}+v_{2}=\left(u_{3} v_{4}-u_{4} v_{3}\right)+\left(u_{5} v_{6}-u_{6} v_{5}\right)+\ldots+\left(u_{2 k-1} v_{2 k}-u_{2 k} v_{2 k-1}\right), \\
& u_{2}+v_{3}=\left(u_{4} v_{5}-u_{5} v_{4}\right)+\left(u_{6} v_{7}-u_{7} v_{6}\right)+\ldots+\left(u_{2 k} v_{2 k+1}-u_{2 k+1} v_{2 k}\right),
\end{aligned}
$$

see Sect. 2.5 for the associated Lax representations.

- One can show that all Monge-Ampère systems of type (2) possess infinitely many hydrodynamic reductions, see [7, 8] for further details.

- In Theorem 4 of Sect. 3 we demonstrate that the necessary and sufficient conditions for a codimension two submanifold $X \subset \mathbf{G r}(d, V)$ to be a linear section is that the only 'essential' second fundamental forms of $X$ are the ones coming from $\mathbf{G r}(d, V)$ itself. This property can be reformulated as a system of second-order differential constraints for the functions $F, G$ defining system (1) thus providing an invariant differential-geometric characterisation of Monge-Ampère systems. 
Remark. In 2D, Monge-Ampère systems (2) constitute a translationally invariant subclass of the more general Jacobi systems,

$$
\begin{aligned}
& a_{1}+b_{1} u_{1}+c_{1} u_{2}+d_{1} v_{1}+e_{1} v_{2}+f_{1}\left(u_{1} v_{2}-u_{2} v_{1}\right)=0 \\
& a_{2}+b_{2} u_{1}+c_{2} u_{2}+d_{2} v_{1}+e_{2} v_{2}+f_{2}\left(u_{1} v_{2}-u_{2} v_{1}\right)=0
\end{aligned}
$$

where the coefficients $a_{i}, b_{i}, c_{i}, d_{i}, e_{i}, f_{i}$ are functions of $x^{1}, x^{2}, u, v$. Geometrically, Jacobi systems are specified by the vanishing of a pair of 2 -forms on a 4 -dimensional manifold with coordinates $x^{1}, x^{2}, u, v$. The theory of such systems was thoroughly developed in [14]. An interesting link of 2D Jacobi systems to the generalised complex geometry of Hitchin is discussed in [1].

The situation in higher dimensions is much more delicate. A Jacobi system for $n$ unknown functions $u^{i}$ of the $n$ independent variables $x^{i}$ is a set of $n$ first-order partial differential equations, each of which is a linear combination of the minors (of all possible orders) of the corresponding $n \times n$ Jacobian matrix. Geometrically, such systems are specified by the vanishing of $n$ differential $n$-forms on the $2 n$ dimensional manifold with coordinates $x^{i}, u^{i}$. Even in the translationally invariant setting, the questions of linearisability/integrability of such systems are largely open.

\section{Classification of Monge-Ampère systems}

\subsection{Jordan-Kronecker normal forms of skew-symmetric pencils}

Here we follow [10] to review Jordan-Kronecker normal forms of skew-symmetric pencils on a vector space $V$ of dimension $d+2$. Any such pencil gives rise to two elements in $\Lambda^{2}(V)$. Taking the dual elements in $\Lambda^{d}\left(V^{*}\right)$ and equating them to zero we obtain normal forms of Monge-Ampère systems.

A skew-symmetric pencil can be written in the form $\mu A+\lambda B$ where $A$ and $B$ are skew-symmetric matrices considered modulo simultaneous transformations $A \rightarrow X A X^{t}, B \rightarrow X B X^{t}$, while $[\lambda: \mu] \in \mathbb{P}^{1}$ is defined modulo automorphisms of $\mathbb{P}^{1}$. Normal forms of such pencils are classified by the following data:

- minimal indices $0 \leq m_{1} \leq m_{2} \leq \cdots \leq m_{p}, \quad p \geq 0$ (in particular, the set of minimal indices can be empty). Each minimal index $m_{i}$ corresponds to a Kronecker block $\mathcal{M}_{m_{i}}$ of the odd size $\left(2 m_{i}+1\right) \times\left(2 m_{i}+1\right)$.

- elementary divisors $\left(a_{1} \mu+b_{1} \lambda\right)^{e_{1}}, \ldots,\left(a_{q} \mu+b_{q} \lambda\right)^{e_{q}}$ where $\left[a_{i}: b_{i}\right]$ are considered as points in $\mathbb{P}^{1}$. Each elementary divisor $\left(a_{i} \mu+b_{i} \lambda\right)^{e_{i}}$ corresponds to a Jordan block $\mathcal{E}_{e_{i}}\left[a_{i}: b_{i}\right]$ of the even size $2 e_{i} \times 2 e_{i}$.

Explicitly, the canonical form of the pencil specified by these data is

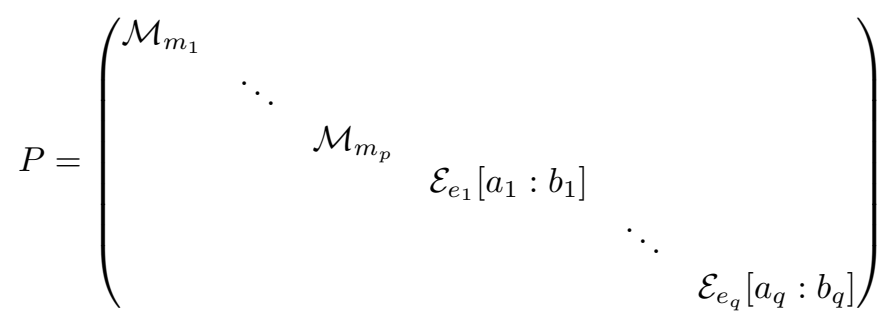


where the Kronecker blocks $\mathcal{M}_{m}$ and the Jordan blocks $\mathcal{E}_{n}[a: b]$ are defined as follows:

$$
\begin{aligned}
\mathcal{M}_{m} & =\left(\begin{array}{cc}
0 & M_{m} \\
-M_{m}^{t} & 0
\end{array}\right), \quad \text { size }(2 m+1) \times(2 m+1), \mathcal{M}_{0}=(0), \\
\mathcal{E}_{n}([1: b]) & =\left(\begin{array}{cc}
0 & E_{n}(b) \\
-E_{n}(b)^{t} & 0
\end{array}\right), \quad \text { size }(2 n) \times(2 n), \\
\mathcal{E}_{n}([0: 1]) & =\left(\begin{array}{cc}
0 & F_{n} \\
-F_{n}^{t} & 0
\end{array}\right), \quad \text { size }(2 n) \times(2 n) .
\end{aligned}
$$

Here we use the notation

$$
\begin{aligned}
& M_{m}=\left(\begin{array}{cccccc} 
& & & & & \lambda \\
& & & \lambda & \mu \\
& & & \cdot & \mu & \\
& & & \cdot & \cdot & \\
& \lambda & \cdot & & & \\
\lambda & \mu & & & & \\
\mu & & & & &
\end{array}\right), \quad \operatorname{size}(m+1) \times m
\end{aligned}
$$

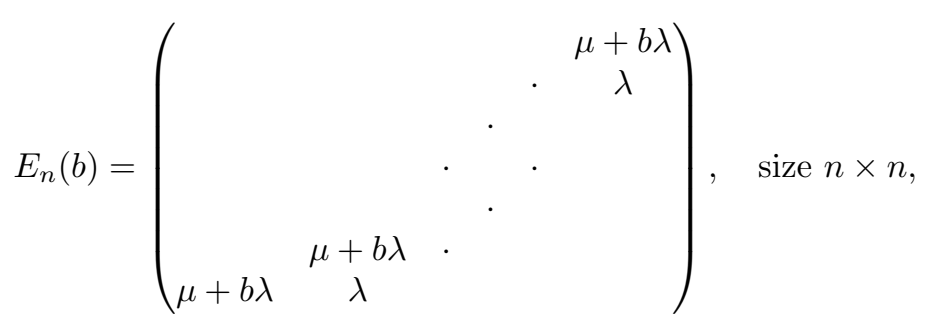

$$
\begin{aligned}
& F_{n}=\left(\begin{array}{ccccc} 
& & & & \lambda \\
& & & \cdot & \mu \\
& & \cdot & & \\
& & \cdot & \cdot & \\
& \lambda & & \cdot & \\
\lambda & \mu & & & \\
\lambda & & &
\end{array}\right), \quad \text { size } n \times n .
\end{aligned}
$$

In addition, elementary divisors are considered up to non-degenerate linear transformations of $\lambda$ and $\mu$, in other words, parameters $\left[a_{i}: b_{i}\right]$ are considered modulo projective transformations. We also impose the following non-degeneracy conditions:

- The pencil does not have zero minimal indices (that is, no $1 \times 1$ zero Kronecker blocks $\mathcal{M}_{0}$ ). Otherwise, the corresponding Monge-Ampère system reduces to a system of lower dimension.

- For $d \geq 3$, the pencil does not contain elements of rank two. These elements correspond to equations of the type $u_{i}=0$ and result in degenerate systems with characteristic varieties of rank 2 .

Any element of rank four in the pencil gives rise to an equation of the type $u_{2}-v_{1}=0$. Introducing the potential $w$ such that $w_{1}=u, w_{2}=v$, we can reduce the corresponding system to a single secondorder Monge-Ampère equation for $w$. Note that a pencil may contain several elements of rank four that might lead to non-equivalent second-order Monge-Ampère equations (see Remark 2 in Sect. 2.3). 


\subsection{Linearisability of Monge-Ampère systems for $d=2,3$}

The classification of $4 \times 4$ and $5 \times 5$ skew-symmetric pencils leads to the following result:

Theorem 1 For $d=2,3$, any non-degenerate system of Monge-Ampère type is linearisable.

\section{Proof:}

For $d=2$ one needs to classify non-degenerate $4 \times 4$ pencils. Note that in this case we allow elements of rank two in the pencil. There are only two non-equivalent normal forms without zero minimal indices, namely

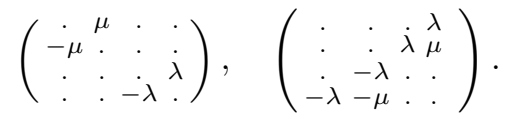

Both pencils give rise to linear systems. Indeed, the first pencil corresponds to 2 -forms

$$
d z^{1} \wedge d z^{2} \text { and } d z^{3} \wedge d z^{4} .
$$

$\left(z^{1}, \ldots, z^{4}\right.$ denote coordinates in 4-dimensional space $\left.V\right)$. Setting $u=z^{4}, v=z^{2}, x^{1}=z^{1}, x^{2}=z^{3}$ and equating these 2 -forms to zero we obtain the linear hyperbolic system $u_{1}=0, v_{2}=0$ (note that we do not need to use the duality transformation for $d=2$ ). Similarly, the second pencil corresponds to 2 -forms

$$
d z^{1} \wedge d z^{4}+d z^{2} \wedge d z^{3} \text { and } d z^{2} \wedge d z^{4}
$$

Setting $u=z^{4}, v=z^{3}, x^{1}=z^{1}, x^{2}=z^{2}$ and equating these 2-forms to zero we obtain the linear parabolic system $u_{1}=0, v_{1}-u_{2}=0$.

For $d=3$ one needs to classify non-degenerate $5 \times 5$ pencils. The non-degeneracy constraints imply that the only possibility is a single $5 \times 5$ Kronecker block,

$$
\left(\begin{array}{ccccc}
\cdot & \cdot & \cdot & \cdot & \lambda \\
\cdot & \cdot & \cdot & \lambda & \mu \\
\cdot & \cdot & \cdot & \mu & \cdot \\
\cdot & -\lambda & -\mu & \cdot & \cdot \\
-\lambda & -\mu & \cdot & \cdot & \cdot
\end{array}\right) .
$$

It is generated by the bi-vectors

$$
\partial_{z^{1}} \wedge \partial_{z^{5}}+\partial_{z^{2}} \wedge \partial_{z^{4}} \text { and } \partial_{z^{2}} \wedge \partial_{z^{5}}+\partial_{z^{3}} \wedge \partial_{z^{4}}
$$

the corresponding dual 3-forms are

$$
d z^{2} \wedge d z^{3} \wedge d z^{4}+d z^{1} \wedge d z^{3} \wedge d z^{5}, \quad d z^{1} \wedge d z^{3} \wedge d z^{4}+d z^{1} \wedge d z^{2} \wedge d z^{5}
$$

Setting $u=z^{5}, v=z^{4}, x^{1}=z^{1}, x^{2}=z^{2}, x^{3}=z^{3}$ and equating these 3 -forms to zero we obtain the linear hyperbolic system $v_{1}-u_{2}=0, v_{2}-u_{3}=0$. This finishes the proof of Theorem 1 .

We emphasize that the linearisability of Monge-Ampère systems for $d=2,3$ does not generalise to higher dimensions $d \geq 4$, see the classification results below.

\subsection{Classification of Monge-Ampère systems for $d=4$}

The classification of $6 \times 6$ skew-symmetric pencils leads to the following result:

Theorem 2 In four dimensions, any non-degenerate system of Monge-Ampère type is $\mathbf{S L}(6)$-equivalent to one of the following normal forms:

1. $u_{2}-v_{1}=0, u_{3}+v_{4}=0$,

2. $u_{2}-v_{1}=0, u_{3}+v_{4}+u_{1} v_{2}-u_{2} v_{1}=0$, 
3. $u_{2}-v_{1}=0, \quad u_{3} v_{4}-u_{4} v_{3}-1=0$,

4. $u_{2}-v_{1}=0, \quad u_{1}+v_{2}+u_{3} v_{4}-u_{4} v_{3}=0$.

\section{Proof:}

One needs to classify non-degenerate $6 \times 6$ skew-symmetric pencils. First assume that there is a non-empty set of minimal indices. As any minimal index of the pencil corresponds to a Kronecker block of odd size, there should be two of them, both equal to 1 . This leads to the normal form consisting of two $3 \times 3$ Kronecker blocks,

$$
\left(\begin{array}{cccccc}
\cdot & \cdot & \lambda & \cdot & \cdot & \cdot \\
\cdot & \cdot & \mu & \cdot & \cdot & \cdot \\
-\lambda & -\mu & \cdot & \cdot & \cdot & \cdot \\
\cdot & \cdot & \cdot & \cdot & \cdot & \lambda \\
\cdot & \cdot & \cdot & \cdot & \cdot & \mu \\
\cdot & \cdot & \cdot & -\lambda & -\mu & \cdot
\end{array}\right)
$$

which corresponds to linear system 1 . Indeed, the above pencil is generated by the bi-vectors

$$
\partial_{z^{1}} \wedge \partial_{z^{3}}+\partial_{z^{4}} \wedge \partial_{z^{6}} \quad \text { and } \quad \partial_{z^{2}} \wedge \partial_{z^{3}}+\partial_{z^{5}} \wedge \partial_{z^{6}}
$$

The corresponding dual 4-forms are

$$
d z^{2} \wedge d z^{4} \wedge d z^{5} \wedge d z^{6}+d z^{1} \wedge d z^{2} \wedge d z^{3} \wedge d z^{5}, \quad d z^{1} \wedge d z^{4} \wedge d z^{5} \wedge d z^{6}+d z^{1} \wedge d z^{2} \wedge d z^{3} \wedge d z^{4} .
$$

Setting $u=z^{6}, v=z^{3}, x^{1}=z^{4}, x^{2}=z^{1}, x^{3}=-z^{2}, x^{4}=z^{5}$ and equating these 4-forms to zero we obtain linear system 1 .

Now assume that the set of minimal indices is empty. The non-degeneracy assumption implies that for any $\left[a_{i}: b_{i}\right]$, there can be only one elementary divisor $\left(a_{i} \mu+b_{i} \lambda\right)^{e_{i}}$. So, up to projective transformations the only possible lists of elementary divisors are:

- $\left\{\lambda^{3}\right\}$,

- $\left\{\lambda^{2}, \mu\right\}$,

- $\{\lambda, \mu, \lambda+\mu\}$.

Explicitly, the associated pencils have the form

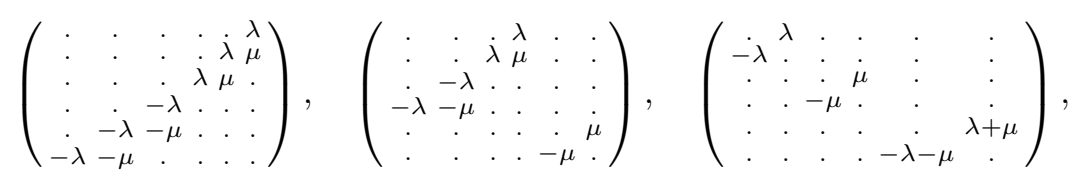

which correspond to systems 2-4, respectively. This finishes the proof of Theorem 2.

Remark 1. It was demonstrated in [12] that self-dual Ricci-flat geometry can be described by the Monge-Ampère system

$$
u_{2}-v_{1}=0, \quad v_{2}+u_{3} v_{4}-u_{4} v_{3}=0,
$$

which, upon the substitution $w_{1}=u, w_{2}=v$, implies the Grant equation $w_{22}+w_{13} w_{24}-w_{14} w_{23}=0$. Note that the linear transformation $x^{2} \rightarrow-v, v \rightarrow x^{2}$ identifies the above system with system 3 from Theorem 2 which corresponds to the first heavenly equation [12].

Remark 2. Let us consider system 3,

$$
u_{2}-v_{1}=0, \quad u_{3} v_{4}-u_{4} v_{3}-1=0
$$


which is related to the first heavenly equation. Interchanging the roles of $u$ and $x^{3}$ we obtain the equivalent system,

$$
u_{3}-v_{4}=0, \quad u_{2}+v_{1} u_{3}-v_{3} u_{1}=0,
$$

which leads to the modified heavenly equation, $w_{24}+w_{13} w_{34}-w_{33} w_{14}=0$, for the potential $w$ defined by the relations $w_{4}=u, w_{3}=v$. The modified heavenly equation appeared recently in the classification of integrable symplectic Monge-Ampère equations [5]. Thus, system 3 provides a Bäcklund transformation connecting the first heavenly and the modified heavenly equations. We point out that these second-order equations are not equivalent under the natural equivalence group $\mathbf{S p}(8)$ acting on symplectic MongeAmpère equations in 4D.

Remark 3. All nonlinear systems from Theorem 2 possess Lax pairs of the form $[X, Y]=0$ where $X$ and $Y$ are parameter-dependent vector fields.

System 2: $u_{2}-v_{1}=0, u_{3}+v_{4}+u_{1} v_{2}-u_{2} v_{1}=0$,

$$
X=\partial_{4}+u_{1} \partial_{2}-u_{2} \partial_{1}+\lambda \partial_{1}, \quad Y=\partial_{3}-v_{1} \partial_{2}+v_{2} \partial_{1}-\lambda \partial_{2} .
$$

System 3: $u_{2}-v_{1}=0, \quad u_{3} v_{4}-u_{4} v_{3}-1=0$,

$$
X=u_{3} \partial_{4}-u_{4} \partial_{3}+\lambda \partial_{1}, \quad Y=-v_{3} \partial_{4}+v_{4} \partial_{3}-\lambda \partial_{2} .
$$

System $4: u_{2}-v_{1}=0, \quad u_{1}+v_{2}+u_{3} v_{4}-u_{4} v_{3}=0$,

$$
X=\partial_{2}+u_{3} \partial_{4}-u_{4} \partial_{3}+\lambda \partial_{1}, \quad Y=\partial_{1}-v_{3} \partial_{4}+v_{4} \partial_{3}-\lambda \partial_{2} .
$$

Modifications of the inverse scattering transform and the $\bar{\partial}$-dressing method for Lax pairs of this type were developed in $[15,16,2]$.

\subsection{Classification of Monge-Ampère systems for $d=5$}

The classification of $7 \times 7$ skew-symmetric pencils leads to the following result:

Theorem 3 In five dimensions, any non-degenerate system of Monge-Ampère type is $\mathbf{S L}(7)$-equivalent to one of the following normal forms:

$$
\begin{aligned}
& \text { 1. } u_{1}+v_{2}+u_{3} v_{4}-u_{4} v_{3}=0, \quad u_{2}+v_{3}+u_{4} v_{5}-u_{5} v_{4}=0, \\
& \text { 2. } u_{2}-v_{1}=0, \quad u_{1}+v_{5}+u_{3} v_{4}-u_{4} v_{3}=0, \\
& \text { 3. } u_{2}-v_{1}=0, \quad u_{4}+v_{5}+u_{1} v_{3}-u_{3} v_{1}=0, \\
& \text { 4. } u_{2}-v_{1}=0, \quad u_{5}+u_{3} v_{4}-u_{4} v_{3}=0 .
\end{aligned}
$$

\section{Proof:}

One needs to classify non-degenerate $7 \times 7$ skew-symmetric pencils. As the size of matrices is odd, the set of minimal indices cannot be empty. Simple analysis shows that there can be at most one minimal index equal to 1,2 or 3 . The latter case is generic and corresponds to the single Kronecker block

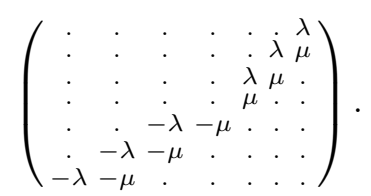

It leads to system 1 . If the minimal index is 2 , then we can assume that the remaining elementary divisor is $\lambda$. If the minimal index is 1 , then the possible lists of minimal divisors are equivalent to $\left\{\lambda^{2}\right\}$ or $\{\lambda, \mu\}$. Explicitly, these three pencils are:

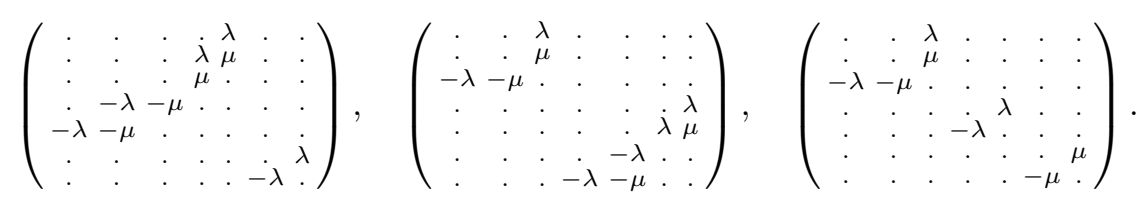


The corresponding systems are 2, 3 and 4, respectively. This finishes the proof of Theorem 3.

Remark. All systems from Theorem 3 possess Lax pairs of the form $[X, Y]=0$ where $X$ and $Y$ are parameter-dependent vector fields.

System 1: $u_{1}+v_{2}+u_{3} v_{4}-u_{4} v_{3}=0, \quad u_{2}+v_{3}+u_{4} v_{5}-u_{5} v_{4}=0$,

$$
\begin{gathered}
X=\partial_{2}+\lambda \partial_{3}+u_{3} \partial_{4}-u_{4} \partial_{3}+\lambda\left(u_{4} \partial_{5}-u_{5} \partial_{4}\right), \\
Y=\partial_{1}+\lambda \partial_{2}-v_{3} \partial_{4}+v_{4} \partial_{3}-\lambda\left(v_{4} \partial_{5}-v_{5} \partial_{4}\right) .
\end{gathered}
$$

System 2: $u_{2}-v_{1}=0, \quad u_{1}+v_{5}+u_{3} v_{4}-u_{4} v_{3}=0$,

$$
X=\partial_{5}+u_{3} \partial_{4}-u_{4} \partial_{3}+\lambda \partial_{1}, \quad Y=\partial_{1}-v_{3} \partial_{4}+v_{4} \partial_{3}-\lambda \partial_{2}
$$

System 3: $u_{2}-v_{1}=0, \quad u_{4}+v_{5}+u_{1} v_{3}-u_{3} v_{1}=0$

$$
X=\partial_{5}+u_{1} \partial_{3}-u_{3} \partial_{1}+\lambda \partial_{1}, \quad Y=\partial_{4}-v_{1} \partial_{3}+v_{3} \partial_{1}-\lambda \partial_{2} .
$$

System 4: $u_{2}-v_{1}=0, \quad u_{5}+u_{3} v_{4}-u_{4} v_{3}=0$,

$$
X=u_{3} \partial_{4}-u_{4} \partial_{3}+\lambda \partial_{1}, \quad Y=\partial_{5}-v_{3} \partial_{4}+v_{4} \partial_{3}-\lambda \partial_{2}
$$

\subsection{Monge-Ampère systems for arbitrary $d$}

Since normal forms of skew-symmetric pencils in even/odd dimensions are essentially different, we will consider these cases separately. Moreover, we will only discuss generic normal forms.

Even dimension. For $d=2 k+2$ a generic skew-symmetric pencil can be brought to the Jordan normal form with $2 \times 2$ blocks along the diagonal. The corresponding system is

$$
\begin{gathered}
u_{2 k+1}=\left(u_{1} v_{2}-u_{2} v_{1}\right)+\left(u_{3} v_{4}-u_{4} v_{3}\right)+\ldots+\left(u_{2 k-1} v_{2 k}-u_{2 k} v_{2 k-1}\right), \\
v_{2 k+2}=a_{1}\left(u_{1} v_{2}-u_{2} v_{1}\right)+a_{2}\left(u_{3} v_{4}-u_{4} v_{3}\right)+\ldots+a_{k}\left(u_{2 k-1} v_{2 k}-u_{2 k} v_{2 k-1}\right),
\end{gathered}
$$

here $a_{1}, \ldots, a_{k}$ are arbitrary constants. Relabelling coordinates we can rewrite these equations in the form

$$
u_{t}=\sum_{i=1}^{k}\left(u_{x^{i}} v_{y^{i}}-u_{y^{i}} v_{x^{i}}\right), \quad v_{\tau}=\sum_{i=1}^{k} a_{i}\left(u_{x^{i}} v_{y^{i}}-u_{y^{i}} v_{x^{i}}\right) .
$$

The corresponding Lax pair is given by

$$
X=\partial_{\tau}+\sum_{i=1}^{k} \alpha_{i}\left(u_{x^{i}} \partial_{y^{i}}-u_{y^{i}} \partial_{x^{i}}\right), \quad Y=\partial_{t}+\sum_{i=1}^{k} \beta_{i}\left(v_{x^{i}} \partial_{y^{i}}-v_{y^{i}} \partial_{x^{i}}\right),
$$

where $\alpha_{i}=\frac{1}{\lambda}-a_{i}, \beta_{i}=1-\lambda a_{i}$.

Odd dimension. For $d=2 k+1$ a generic skew-symmetric pencil can be brought to the Kronecker normal form. The corresponding system is

$$
\begin{aligned}
& u_{1}+v_{2}=\left(u_{3} v_{4}-u_{4} v_{3}\right)+\left(u_{5} v_{6}-u_{6} v_{5}\right)+\ldots+\left(u_{2 k-1} v_{2 k}-u_{2 k} v_{2 k-1}\right) \\
& u_{2}+v_{3}=\left(u_{4} v_{5}-u_{5} v_{4}\right)+\left(u_{6} v_{7}-u_{7} v_{6}\right)+\ldots+\left(u_{2 k} v_{2 k+1}-u_{2 k+1} v_{2 k}\right)
\end{aligned}
$$

Its Lax pair is given by

$$
X=\partial_{2}+\lambda \partial_{3}-\sum_{i=3}^{2 k} \alpha_{i}\left(u_{i} \partial_{i+1}-u_{i+1} \partial_{i}\right), \quad Y=\partial_{1}+\lambda \partial_{2}+\sum_{i=3}^{2 k} \alpha_{i}\left(v_{i} \partial_{i+1}-v_{i+1} \partial_{i}\right),
$$

where $\alpha_{2 s-1}=1, \alpha_{2 s}=\lambda$.

Since generic normal forms are integrable in any dimension, and integrability is preserved in the limit, we conclude that all systems of Monge-Ampère type must be integrable. 


\section{Differential geometry of Monge-Ampère systems}

Consider system (1) of dimension $d=m+1$. Representing it in evolutionary form,

$$
u_{m+1}=f\left(u_{1}, \ldots, u_{m}, v_{1}, \ldots, v_{m}\right), \quad v_{m+1}=g\left(u_{1}, \ldots, u_{m}, v_{1}, \ldots, v_{m}\right),
$$

we will derive differential constraints for the functions $f$ and $g$ that characterise systems (2) of MongeAmpère type. Let us begin with the simplest case $d=2$,

$$
u_{2}=f\left(u_{1}, v_{1}\right), \quad v_{2}=g\left(u_{1}, v_{1}\right)
$$

which however contains all essential ingredients of the general case.

Proposition 1. System (6) is of Monge-Ampère type if and only if the (symmetric) differentials $d^{2} f$ and $d^{2} g$ are proportional to the quadratic form $d f d v_{1}-d g d u_{1}$ :

$$
d^{2} f, d^{2} g \in \operatorname{span}\left\{d f d v_{1}-d g d u_{1}\right\}
$$

\section{Proof:}

Equations (6) specify a surface $X$ in the Grassmannian $\mathbf{G r}(2,4)$. The Plücker embedding of $\mathbf{G r}(2,4)$ into $\mathbb{P}^{5}$ is a quadric with position vector $\left(u_{1}, v_{1}, u_{2}, v_{2}, u_{2} v_{1}-u_{1} v_{2}\right)$. The induced embedding of $X$ has position vector

$$
R=\left(u_{1}, v_{1}, f, g, v_{1} f-u_{1} g\right) .
$$

To prove that system (6) is of Monge-Ampère type we need to show that components of $R$ satisfy 2 linear relations with constant coefficients or, equivalently, that the Plücker image of $X$ lies in a 3-dimensional linear subspace of $\mathbb{P}^{5}$. This means that the union of all osculating spaces of $X$ must be 3-dimensional. Since the tangent space of $X$, spanned by the vectors

$$
\begin{aligned}
& R_{u_{1}}=\left(1,0, f_{u_{1}}, g_{u_{1}}, v_{1} f_{u_{1}}-u_{1} g_{u_{1}}-g\right), \\
& R_{v_{1}}=\left(0,1, f_{v_{1}}, g_{v_{1}}, v_{1} f_{v_{1}}-u_{1} g_{v_{1}}+f\right),
\end{aligned}
$$

is already 2-dimensional, we have to show that the union of the second- and third-order osculating spaces (spanned by the second- and third-order partial derivatives of the position vector $R$ with respect to $u_{1}$ and $v_{1}$ ) has dimension 1 . As higher-order derivatives of $R$ have zeros in the first two positions, the rank of the following matrix must equal 1 :

$$
r k\left(\begin{array}{ccc}
f_{u_{1} u_{1}} & g_{u_{1} u_{1}} & v_{1} f_{u_{1} u_{1}}-u_{1} g_{u_{1} u_{1}}-2 g_{u_{1}} \\
f_{u_{1} v_{1}} & g_{u_{1} v_{1}} & v_{1} f_{u_{1} v_{1}}-u_{1} g_{u_{1} v_{1}}+f_{u_{1}}-g_{v_{1}} \\
f_{v_{1} v_{1}} & g_{v_{1} v_{1}} & v_{1} f_{v_{1} v_{1}}-u_{1} g_{v_{1} v_{1}}+2 f_{v_{1}} \\
f_{u_{1} u_{1} u_{1}} & g_{u_{1} u_{1} u_{1}} & v_{1} f_{u_{1} u_{1} u_{1}}-u_{1} g_{u_{1} u_{1} u_{1}}-3 g_{u_{1}} \\
f_{u_{1} u_{1} v_{1}} & g_{u_{1} u_{1} v_{1}} & v_{1} f_{u_{1} u_{1} v_{1}}-u_{1} g_{u_{1} u_{1} v_{1}}+f_{u_{1} u_{1}}-2 g_{u_{1} v_{1}} \\
f_{u_{1} v_{1} v_{1}} & g_{u_{1} v_{1} v_{1}} & v_{1} f_{u_{1} v_{1} v_{1}}-u_{1} g_{u_{1} v_{1} v_{1}}+2 f_{u_{1} v_{1}}-g_{v_{1} v_{1}} \\
f_{v_{1} v_{1} v_{1}} & g_{v_{1} v_{1} v_{1}} & v_{1} f_{v_{1} v_{1} v_{1}}-u_{1} g_{v_{1} v_{1} v_{1}}+3 f_{v_{1} v_{1}}
\end{array}\right)=1 .
$$

Since the terms of the third column containing multiples of $v_{1}$ or $u_{1}$ are proportional to the first and second columns, respectively, and can therefore be eliminated without changing the rank, we obtain a simpler condition,

$$
r k\left(\begin{array}{ccc}
f_{u_{1} u_{1}} & g_{u_{1} u_{1}} & -2 g_{u_{1}} \\
f_{u_{1} v_{1}} & g_{u_{1} v_{1}} & f_{u_{1}}-g_{v_{1}} \\
f_{v_{1} v_{1}} & g_{v_{1} v_{1}} & 2 f_{v_{1}} \\
f_{u_{1} u_{1} u_{1}} & g_{u_{1} u_{1} u_{1}} & -3 g_{u_{1}} \\
f_{u_{1} u_{1} v_{1}} & g_{u_{1} u_{1} v_{1}} & f_{u_{1} u_{1}}-2 g_{u_{1} v_{1}} \\
f_{u_{1} v_{1} v_{1}} & g_{u_{1} v_{1} v_{1}} & 2 f_{u_{1} v_{1}}-g_{v_{1} v_{1}} \\
f_{v_{1} v_{1} v_{1}} & g_{v_{1} v_{1} v_{1}} & 3 f_{v_{1} v_{1}}
\end{array}\right)=1
$$


This condition is equivalent to the requirement that the first and second columns are proportional to the third column. Let $p$ and $q$ be the corresponding coefficients of proportionality. In compact form, this can be represented as

$$
d^{2} f=2 p\left(d f d v_{1}-d g d u_{1}\right), \quad d^{2} g=2 q\left(d f d v_{1}-d g d u_{1}\right),
$$

and

$$
d^{3} f=3 p\left(d^{2} f d v_{1}-d^{2} g d u_{1}\right), \quad d^{3} g=3 q\left(d^{2} f d v_{1}-d^{2} g d u_{1}\right)
$$

respectively. Calculating (symmetric) differentials of (8) and comparing the result with (9) we obtain the equations for $p$ and $q$,

$$
d p=p\left(p d v_{1}-q d u_{1}\right), \quad d q=q\left(p d v_{1}-q d u_{1}\right) .
$$

Equations (8) and (10) constitute a closed involutive differential system for $f$ and $g$ which characterises Monge-Ampère systems. It remains to note that conditions (10) can be obtained as the consistency conditions of equations (8) alone, without using (9). In other words, equations (8) imply both (9) and (10). This finishes the proof of Proposition 1.

Remark 1. Condition (7) has a clear projective-geometric interpretation. Recall that the second fundamental forms of $X \subset \mathbb{P}^{5}$ are spanned by $d^{2} f, d^{2} g$ and $d f d v_{1}-d g d u_{1}$. Here the last form is the restriction to $X$ of the second fundamental form of the Grassmannian $\mathbf{G r}(2,4)$, namely, $d u_{2} d v_{1}-d v_{2} d u_{1}$. Thus, (7) says that the only 'essential' second fundamental form of $X \subset \mathbf{G r}(2,4)$ is the one coming from the second fundamental form of $\operatorname{Gr}(2,4) \subset \mathbb{P}^{5}$. This property is clearly necessary for $X$ to be a linear section. The above result shows that in this particular case it is also sufficient.

Remark 2. Condition (7) can be written as a system of PDEs for $f$ and $g$, indeed, the elimination of $p$ and $q$ from (8) implies the second-order relations

$$
\begin{aligned}
& f_{u_{1} u_{1}}=\frac{2 g_{u_{1}}}{g_{v_{1}}-f_{u_{1}}} f_{u_{1} v_{1}}, \quad f_{v_{1} v_{1}}=\frac{2 f_{v_{1}}}{f_{u_{1}}-g_{v_{1}}} f_{u_{1} v_{1}}, \\
& g_{u_{1} u_{1}}=\frac{2 g_{u_{1}}}{g_{v_{1}}-f_{u_{1}}} g_{u_{1} v_{1}}, \quad g_{v_{1} v_{1}}=\frac{2 f_{v_{1}}}{f_{u_{1}}-g_{v_{1}}} g_{u_{1} v_{1}} .
\end{aligned}
$$

The case of arbitrary dimension $d=m+1$ can be considered in a similar way.

Theorem 4 System (5) is of Monge-Ampère type if and only if

$$
d^{2} f, d^{2} g \in \operatorname{span}\left\{d u_{i} d v_{j}-d u_{j} d v_{i}, d f d v_{i}-d g d u_{i} \mid i, j=1, \ldots, m\right\} .
$$

\section{Proof:}

Equations (5) specify a submanifold $X$ in $\mathbf{G r}(d, V)$ whose Plücker embedding into $\mathbb{P} \Lambda^{2}(V)$ has position vector

$$
\left(u_{i}, v_{i}, f, g, u_{i} v_{j}-u_{j} v_{i}, v_{i} f-u_{i} g\right), \quad i, j=1, \ldots, m
$$

To prove that system (5) is of Monge-Ampère type we need to show that $X$ lies in a linear subspace of codimension two. Calculation of osculating spaces similar to that from the proof of Proposition 1 implies that this requirement is equivalent to the conditions

$$
\begin{aligned}
& d^{2} f=2 a^{i j}\left(d u_{i} d v_{j}-d u_{j} d v_{i}\right)+2 p^{i}\left(d f d v_{i}-d g d u_{i}\right) \\
& d^{2} g=2 b^{i j}\left(d u_{i} d v_{j}-d u_{j} d v_{i}\right)+2 q^{i}\left(d f d v_{i}-d g d u_{i}\right)
\end{aligned}
$$

as well as

$$
d^{3} f=3 p^{i}\left(d^{2} f d v_{i}-d^{2} g d u_{i}\right), \quad d^{3} g=3 q^{i}\left(d^{2} f d v_{i}-d^{2} g d u_{i}\right),
$$

(the standard summation convention is assumed). Moreover, for the first two terms in (13) we assume $i<j$. Calculating (symmetric) differentials of (13) and comparing the result with (14) we obtain the equations for the coefficients,

$$
\begin{gathered}
d a^{i j}=a^{i j} \omega_{1}-b^{i j} \omega_{2}, \quad d b^{i j}=a^{i j} \varphi_{1}-b^{i j} \varphi_{2}, \\
d p^{i}=p^{i} \omega_{1}-q^{i} \omega_{2}, \quad d q^{i}=p^{i} \varphi_{1}-q^{i} \varphi_{2}
\end{gathered}
$$


where we adopt the notation

$$
\omega_{1}=p^{i} d v_{i}, \quad \omega_{2}=p^{i} d u_{i}, \quad \varphi_{1}=q^{i} d v_{i}, \quad \varphi_{2}=q^{i} d u_{i}
$$

We point out that, modulo (15), these forms satisfy the structure equations

$$
d \omega_{1}=\varphi_{1} \wedge \omega_{2}, \quad d \omega_{2}=\omega_{1} \wedge \omega_{2}+\varphi_{2} \wedge \omega_{2}, \quad d \varphi_{1}=\varphi_{1} \wedge \varphi_{2}+\varphi_{1} \wedge \omega_{1}, \quad d \varphi_{2}=\varphi_{1} \wedge \omega_{2} .
$$

Equations (13) and (15) constitute a closed involutive differential system for $f$ and $g$ which characterises Monge-Ampère systems. It remains to point out that conditions (15) can be obtained as the consistency conditions of equations (13) alone, without using (14). In other words, equations (13) imply both (14) and (15). This finishes the proof of Theorem 4.

Remark 3. Condition (12) means that the only essential second fundamental forms of the submanifold $X \subset \mathbf{G r}(d, V)$ are the ones coming from the Grassmannian itself. These conditions can be written as a system of second-order PDEs for $f$ and $g$,

$$
\begin{gathered}
f_{u_{i} u_{i}}=\frac{2 g_{u_{i}}}{g_{v_{i}}-f_{u_{i}}} f_{u_{i} v_{i}}, \quad f_{v_{i} v_{i}}=\frac{2 f_{v_{i}}}{f_{u_{i}}-g_{v_{i}}} f_{u_{i} v_{i}} \\
f_{u_{i} u_{j}}=\frac{g_{u_{j}}}{g_{v_{i}}-f_{u_{i}}} f_{u_{i} v_{i}}+\frac{g_{u_{i}}}{g_{v_{j}}-f_{u_{j}}} f_{u_{j} v_{j}}, \quad f_{v_{i} v_{j}}=\frac{f_{v_{j}}}{f_{u_{i}}-g_{v_{i}}} f_{u_{i} v_{i}}+\frac{f_{v_{i}}}{f_{u_{j}}-g_{v_{j}}} f_{u_{j} v_{j}} \\
f_{u_{i} v_{j}}+f_{u_{j} v_{i}}=\frac{f_{u_{j}}-g_{v_{j}}}{f_{u_{i}}-g_{v_{i}}} f_{u_{i} v_{i}}+\frac{f_{u_{i}}-g_{v_{i}}}{f_{u_{j}}-g_{v_{j}}} f_{u_{j} v_{j}}
\end{gathered}
$$

here $i, j$ take any values from 1 to $\mathrm{m}$; the equations for $g$ can be obtained by the simultaneous substitution $f \leftrightarrow g$ and $u \leftrightarrow v$. For $m=1$ these conditions reduce to (11).

Remark 4. Each equation (16) involves maximum two distinct indices, namely $i$ and $j$. Thus, if all traveling wave reductions of system (5) to $3 \mathrm{D}$ obtained by setting $u_{k}=$ const, $v_{k}=$ const, $k \neq i, j$, are of Monge-Ampère type, then the full multi-dimensional system (5) must be of Monge-Ampère type as well. This result can be reformulated geometrically as follows. Let $X$ be a codimension two submanifold in $\mathbf{G r}(d, V)$. Suppose that the intersection of $X$ with every $\mathbf{G r}(3,5) \subset \mathbf{G r}(d, V)$ is a linear section of $\mathbf{G r}(3,5)$. Then $X$ itself must be a linear section.

\section{Concluding remarks}

In this paper we have classified two-component systems of Monge-Ampère type and established their integrability in all spacial dimensions. It would be interesting to generalise these results to the multicomponent case. Let $\mathbf{u}=\left(u^{1}(\mathbf{x}), \ldots, u^{n}(\mathbf{x})\right), n \geq 3$, be functions of $d$ independent variables $\mathbf{x}=$ $\left(x^{1}, \ldots, x^{d}\right)$. Consider a first-order Monge-Ampère system

$$
F^{1}\left(\mathbf{u}_{1}, \ldots, \mathbf{u}_{d}\right)=0, \ldots, F^{n}\left(\mathbf{u}_{1}, \ldots, \mathbf{u}_{d}\right)=0
$$

where each $F^{i}$ is a linear combination of minors of the $n \times d$ Jacobian matrix of $\mathbf{u}(\mathbf{x})$. Geometrically, such systems correspond to sections of $\mathbf{G r}\left(d, V^{n+d}\right)$ by linear spaces of codimension $n$. Based on the present paper and the results of $[5,9]$ we can formulate the following conjectures.

- For $d=3$, the integrability of a Monge-Ampère system is equivalent to its linearisability (which is equivalent to the property that the corresponding linear space of codimension $n$ is tangential to $\left.\operatorname{Gr}\left(3, V^{n+3}\right)\right)$.

- For $d \geq 4$, the integrability of a Monge-Ampère system (for $n \geq 3$ it will no longer be automatic) is equivalent to the property that the corresponding linear space of codimension $n$ is tangential to $\mathbf{G r}\left(d, V^{n+d}\right)$ along a submanifold which meets every $\mathbf{G r}\left(3, V^{n+3}\right) \subset \mathbf{G r}\left(d, V^{n+d}\right)$. 
Another possible line of research is the study of two-component Monge-Ampère systems (Jacobi systems) whose coefficients are arbitrary functions of $x^{1}, \ldots, x^{d}, u, v$. Such systems can be defined by the vanishing of 2 differential $d$-forms on the $(d+2)$-dimensional manifold with coordinates $x^{1}, \ldots, x^{d}, u, v$. By duality, they correspond to a pair of skew-symmetric bivectors. It would be interesting to understand whether there is a relation between integrability in the sense of this paper and bi-Poisson geometry of Turiel [20] and Gelfand-Zakharevich [11].

We hope to return to these questions elsewhere.

\section{Acknowledgements}

We thank the LMS for their support of BD to Loughborough making this collaboration possible. We also thank the referee for useful suggestions.

\section{References}

[1] B. Banos, Monge-Ampère equations and generalized complex geometry - the two-dimensional case, J. Geom. Phys. 57, no. 3 (2007) 841-853.

[2] L.V. Bogdanov and B.G. Konopelchenko, On the $\bar{\partial}$-dressing method applicable to heavenly equation. Phys. Lett. A 345, no. 1-3 (2005) 137-143.

[3] G. Boillat, Sur la forme générale du système de Monge-Ampère, C. R. Acad. Sci. Paris Sér. I Math. 325, no. 3 (1997) 339-342.

[4] C.P. Boyer and J.D. Finley, Killing vectors in self-dual Euclidean Einstein spaces, J. Math. Phys. 23 (1982) 1126-1130.

[5] B. Doubrov and E.V. Ferapontov, On the integrability of symplectic Monge-Ampère equations, Journal of Geometry and Physics 60 (2010) 1604-1616.

[6] B. Doubrov, E.V. Ferapontov, B. Kruglikov, V. Novikov, On integrability in Grassmann geometries: integrable systems associated with fourfolds in $\operatorname{Gr}(3,5)$, arXiv:1503.02274v2.

[7] E.V. Ferapontov and M.V. Pavlov, Hydrodynamic reductions of the heavenly equation, Class. Quantum Grav. 20 (2003) 2429-2441.

[8] E.V. Ferapontov and K.R. Khusnutdinova, Hydrodynamic reductions of multi-dimensional dispersionless PDEs: the test for integrability, J. Math. Phys. 45 (2004) 2365-2377.

[9] E.V. Ferapontov, L. Hadjikos and K.R. Khusnutdinova, Integrable equations of the dispersionless Hirota type and hypersurfaces in the Lagrangian Grassmannian, International Mathematics Research Notices, (2010) 496-535; doi:10.1093/imrn/rnp134.

[10] M.A. Gauger, On the classification of metabelian Lie algebras, Trans. Amer. Math. Soc. 179 (1973) 293-329.

[11] I.M. Gelfand and I. Zakharevich, Webs, Veronese curves, and bi-Hamiltonian systems, J. Funct. Anal. 99, no. 1 (1991) 150-178.

[12] J.D.E. Grant, On self-dual gravity, Phys. Rev. D 47 (1993) 2606-2612.

[13] V. Husain, Self-dual gravity as a two-dimensional theory and conservation laws, Classical Quantum Gravity 11, no. 4 (1994) 927-937.

[14] A. Kushner, V. Lychagin, V. Rubtsov, Contact geometry and non-linear differential equations. Encyclopedia of Mathematics and its Applications, 101. Cambridge University Press, Cambridge (2007), 496 pp. 
[15] S.V. Manakov and P.M. Santini, Inverse scattering problem for vector fields and the Cauchy problem for the heavenly equation, Phys. Lett. A 359, no 6 (2006) 613-619.

[16] S.V. Manakov and P.M. Santini, On the solutions of the second heavenly and Pavlov equations, J. Phys. A 42, no. 40 (2009) 404013, 11 pp.

[17] J.F. Plebański, Some solutions of complex Einstein equations, J. Math. Phys. 16 (1975) 2395-2402.

[18] J.F. Plebański and M. Przanowski, The Lagrangian for a self-dual gravitational field as a limit of the SDYM Lagrangian, Phys. lett. A 212 (1996) 22-28.

[19] K. Takasaki, An infinite number of hidden variables in hyper-Kähler metrics, J. Math. Phys. 30, no. 7 (1989) 1515-1521.

[20] F-J. Turiel, Classification locale simultanée de deux formes symplectiques compatibles, Manuscripta Math. 82, no. 3-4 (1994) 349-362. 